CERN-TH/96-181

hep-ph/9607318

\title{
NLO calculations in QCD: a general algorithm
}

\author{
S. Catani \\ I.N.F.N., Sezione di Firenze \\ and Dipartimento di Fisica, Università di Firenze \\ Largo E. Fermi 2, I-50125 Florence, Italy \\ M.H. Seymour \\ Theory Division, CERN \\ CH-1211 Geneva 23, Switzerland
}

\begin{abstract}
We briefly describe a new general algorithm for carrying out QCD calculations to next-toleading order in perturbation theory. The algorithm can be used for computing arbitrary jet cross sections in arbitrary processes and can be straightforwardly implemented in generalpurpose Monte Carlo programs.
\end{abstract}

CERN-TH/96-181

July 1996

*Invited talk presented by S. Catani at the DESY-Zeuthen Workshop "QCD and QED in Higher Orders", Rheinsberg, Germany, April 1996. To appear in the Proceedings. 


\title{
NLO calculations in QCD: a general algorithm *
}

\author{
S. Catani ${ }^{a}$ and M.H. Seymour ${ }^{b}$ \\ ${ }^{a}$ I.N.F.N., Sezione di Firenze, and Dipartimento di Fisica, Università di Firenze, \\ Largo E. Fermi 2, I-50125 Florence, Italy \\ bTheory Division, CERN, \\ CH-1211 Geneva 23, Switzerland
}

We briefly describe a new general algorithm for carrying out QCD calculations to next-to-leading order in perturbation theory. The algorithm can be used for computing arbitrary jet cross sections in arbitrary processes and can be straightforwardly implemented in general-purpose Monte Carlo programs.

\section{MOTIVATIONS}

During the last fifteen years many efforts have been devoted to carry out accurate QCD calculations to higher perturbative orders. These calculations are motivated by three main reasons.

First of all, the comparison between perturbative calculations and experimental data allow one to perform precision tests of QCD in the stronginteraction processes that involve a large transferred momentum $Q$ [1]. These tests are essential for measuring the strong coupling $\alpha_{\mathrm{S}}(Q)$ and its running as predicted by asymptotic freedom. Perturbative QCD studies are also important to evaluate the background for new physics signals [2]. More recently, a renewed interest in perturbative calculations has been motivated by phenomenological and theoretical models of non-perturbative phenomena [3]. Using these models and having under control the perturbative component, one can use experimental data on high-energy cross sections to extract information on the underlying non-perturbative dynamics.

To these aims, calculations at the leading order (LO) of the perturbative expansion in the QCD coupling $\alpha_{\mathrm{S}}(Q)$ are insufficient. In fact, just because of its perturbative nature, the running of the QCD coupling can be hidden in higherorder corrections by the replacement $\alpha_{\mathrm{S}}(Q)=$

\footnotetext{
* Research supported in part by EEC Programme 'Human Capital and Mobility', Network 'Physics at High Energy Colliders', contract CHRX-CT93-0357 (DG 12 COMA).
}

$\alpha_{\mathrm{S}}^{(0)}\left[1+K(Q) \alpha_{\mathrm{S}}(Q)+\ldots\right], \alpha_{\mathrm{S}}^{(0)}$ being the values of $\alpha_{\mathrm{S}}$ at a fixed (and arbitrary) momentum scale. It follows that a $\mathrm{LO}$ calculation predicts only the order of magnitude of a given cross section and the rough features of a certain observable. The accuracy of the perturbative QCD expansion is instead controlled by the size of the higher-order contributions. Any definite perturbative QCD prediction thus requires (at least) a next-to-leading order (NLO) calculation.

In general, NLO calculations are highly nontrivial. The first bottleneck one encounters in producing new NLO calculations for a certain process is the evaluation of the relevant matrix elements (recent progress in these computations is reviewed in Ref. (4). However, even when the process-dependent matrix elements are available, there are practical difficulties in setting up a straightforward calculational procedure. The physical origin of these difficulties is in the necessity of factorizing the long- and short-distance components of the scattering processes and is reflected in the perturbative expansion by the presence of divergences. QCD theorems guarantee that these divergences eventually cancel in the evaluation of physical cross sections but do not prevent their appearance in intermediate steps. Since single intermediate expressions are usually divergent, the numerical implementation of NLO calculations forms a second bottleneck.

The main issue one has to face is thus the following. On one side many different NLO calcula- 
tions (i.e. calculations for different observables) for a certain process and, possibly, for many processes are warranted. On the other side each calculation is very complicated (see also Sect. 2).

In particular, it is very important to reduce the second bottleneck mentioned above by setting up an efficient and simple method for computing arbitrary quantities in a single process. It would be even more important to have at our disposal a simple algorithm for computing arbitrary quantities in arbitrary processes. The goal is a universal algorithm that, in principle, can be used to construct a general-purpose Monte Carlo program (not a Monte Carlo event generator) for carrying out NLO QCD calculations. Conceptually, such an algorithm could be used in the same manner as some universal Monte Carlo event generators (e.g. HERWIG [5]): any time one wants to compute a new quantity or to vary the experimental cuts, one simply modifies the 'user routine' accordingly; any time one wants to study a different process, one simply enters the corresponding matrix elements.

In this contribution we briefly describe a general algorithm [6] of this type, which is based on the subtraction method and the dipole formalism.

\section{NLO QCD CALCULATIONS}

The general structure of a QCD cross section in NLO is the following

$\sigma=\sigma^{L O}+\sigma^{N L O}$.

Here the LO cross section $\sigma^{L O}$ is obtained by integrating the fully exclusive cross section $d \sigma^{B}$ in the Born approximation over the phase space for the corresponding jet quantity. Let us suppose that this LO calculation involves $m$ partons with momenta $p_{k}(k=1, \ldots, m)$ in the final state. Thus, we write

$\sigma^{L O}=\int_{m} d \sigma^{B}$,

where the Born-level cross section is:

$d \sigma^{B}=d \Phi^{(m)}\left(\left\{p_{k}\right\}\right)\left|\mathcal{M}_{m}\left(\left\{p_{k}\right\}\right)\right|^{2} F_{J}^{(m)}\left(\left\{p_{k}\right\}\right)$

and $d \Phi^{(m)}$ and $\mathcal{M}_{m}$ respectively denote the full phase space and the tree-level QCD matrix ele- ment to produce $m$ final-state partons. These are the factors that depend on the process.

The function $F_{J}^{(m)}$ defines the physical quantity that we want to compute, possibly including the experimental cuts. Note that this quantity has to be a jet observable, that is, it has to be infrared and collinear safe: its actual value has to be independent of the number of soft and collinear particles in the final state. Thus, we should have (we refer to [6] for a more detailed formal definition)

$F_{J}^{(m+1)} \rightarrow F_{J}^{(m)}$,

in any case where the $m+1$-parton configuration on the left-hand side is obtained from the $m$-parton configuration on the right-hand side by adding a soft parton or replacing a parton with a pair of collinear partons carrying the same total momentum.

Efficient techniques, based on helicity amplitudes [7] and colour subamplitude decomposition [8], are available for calculating tree-level matrix elements. Thus the evaluation of the LO cross section does not present any particular difficulty. Even if $\sigma^{L O}$ cannot be computed analytically (because $\mathcal{M}_{m}$ is too cumbersome or the phase-space cuts in $F_{J}^{(m)}$ are very involved), one can straightforwardly use numerical integration techniques, for instance, a Monte Carlo program where the function $F_{J}^{(m)}$ is given as 'user routine'.

At NLO one has to consider the exclusive cross section $d \sigma^{R}$ with $m+1$ partons in the final state and the one-loop correction $d \sigma^{V}$ to the process with $m$ partons in the final state:

$\sigma^{N L O}=\int_{m+1} d \sigma^{R}+\int_{m} d \sigma^{V}$.

The exclusive cross sections $d \sigma^{R}$ and $d \sigma^{V}$ have the same structure as the Born-level cross section in Eq. (3), apart from the replacements $\left|\mathcal{M}_{m}\right|^{2} \rightarrow\left|\mathcal{M}_{m+1}\right|^{2}$ and $\left|\mathcal{M}_{m}\right|^{2} \rightarrow\left|\mathcal{M}_{m}\right|_{(1-\text { loop })}^{2}$. Here $\left|\mathcal{M}_{m}\right|_{(1-\text { loop })}^{2}$ denotes the QCD amplitude to produce $m$ final-state partons evaluated in the one-loop approximation.

The calculation of the loop integral in $\left|\mathcal{M}_{m}\right|_{(1-\text { loop })}^{2}$ leads to ultraviolet, soft and 
collinear singularities. The ultraviolet singularities can be handled in a simple way within the loop corrections by carrying out the renormalization procedure. Thus we can assume that the virtual cross section in Eq. (5) is given in terms of the renormalized matrix element and the ultraviolet divergences have been removed.

Soft and collinear singularities instead lead to the main problem. These singularities do not cancel within the sole $d \sigma^{V}$ and are accompanied by analogous singularities arising from the integration of the real cross section $d \sigma^{R}$. In the case of jet quantities, adding the real and virtual contribution, these singularities cancel and the physical NLO cross section in Eq. (5) is finite. This cancellation is guaranteed by the property in Eq. (何) However, the cancellation mechanism is not trivial because it does not take place at the integrand level.

The two integrals on the right-hand side of Eq. (5) are separately divergent so that, before any numerical calculation can be attempted, the separate pieces have to be regularized. The most widely used regularization procedure (actually, the only regularization procedure that is gauge invariant and Lorentz invariant to any order of the QCD perturbative expansion) is obtained by means of analytic continuation in a number of space-time dimensions $d=4-2 \epsilon$ different from four. Using dimensional regularization, the divergences (arising out of the integration) are replaced by double (soft and collinear) poles $1 / \epsilon^{2}$ and single (soft or collinear) poles $1 / \epsilon$. Thus the real and virtual contributions should be calculated independently, yielding equal-and-opposite poles in $\epsilon$. These poles have to be combined and after having achieved their cancellation the limit $\epsilon \rightarrow 0$ can be safely carried out.

In principle this computation procedure does not pose any problems. In practice, that is not the case. On one side, analytic calculations are impossible for all but the simplest quantities because of the involved kinematics for multi-parton configurations and of the complicated phasespace cuts relative to the definition of the jet observable. On the other side, the use of numerical methods is far from trivial because real and virtual contributions have to be integrated sepa- rately over different phase-space regions and because of the analytic continuation in the arbitrary number $d$ of space-time dimensions.

The most efficient solution to this practical problem consists in using a hybrid analyti$\mathrm{cal} /$ numerical procedure: one must somehow simplify and extract the singular parts of the cross section and treat them analytically; the remainder is treated numerically, independently of the full complications of the jet quantity and of the process.

There are, broadly speaking, two general methods for doing that: the phase-space slicing method and the subtraction method. Both the slicing [9] and the subtraction [10] methods were first used in the context of NLO calculations of three-jet cross sections in $e^{+} e^{-}$annihilation. Then they have been applied to other cross sections, adapting the method each time to the particular process. Only recently has it become clear that both methods are generalizable in a processindependent manner. The key observation is that the singular parts of the QCD matrix elements for real emission can be singled out in a general way by using the factorization properties of soft and collinear radiation [11]. Owing to this universality, the two methods have led to general algorithms for NLO QCD calculations.

In the context of the phase-space slicing method, an algorithm has been developed for jet cross sections in lepton and hadron collisions [12,13]. The complete generalization of this method to include fragmentation functions and heavy flavours is in progress 14,15].

As for the subtraction method, two approaches are available for setting up general algorithms. The 'residue approach' introduced in Ref. [16] has been further generalized in Refs. 117 19 and is discussed elsewhere in these Proceedings [20]. In the rest of this contribution we describe the more recent approach, based on the dipole formalism [6,21. 


\section{THE SUBTRACTION METHOD}

The general idea of the subtraction method is to use the identity

$$
\begin{aligned}
\sigma^{N L O} & =\int_{m+1}\left[d \sigma^{R}-d \sigma^{A}\right] \\
& +\int_{m+1} d \sigma^{A}+\int_{m} d \sigma^{V},
\end{aligned}
$$

which is obtained by subtracting and adding back the same quantity $d \sigma^{A}$. The cross section contribution $d \sigma^{A}$ has to fulfil two main properties.

i) Firstly, it must be a proper approximation of $d \sigma^{R}$ such as to have the same pointwise singular behaviour (in $d$ dimensions) as $d \sigma^{R}$ itself. Thus, $d \sigma^{A}$ acts as a local counterterm for $d \sigma^{R}$ and one can safely perform the limit $\epsilon \rightarrow 0$ under the integral sign in the first term on the right-hand side of Eq. (6). This defines a cross section contribution $\sigma^{N L O}\{m+1\}$ with $m+1$-parton kinematics that can be integrated numerically in four dimensions:

$\sigma^{N L O\{m+1\}}=\int_{m+1}\left[\left(d \sigma^{R}\right)_{\epsilon=0}-\left(d \sigma^{A}\right)_{\epsilon=0}\right]$.

ii) The second property of $d \sigma^{A}$ is its analytic integrability (in $d$ dimensions) over the oneparton subspace leading to the soft and collinear divergences. In this case, we can rewrite the last two terms on the right-hand side of Eq. (6) as follows

$\sigma^{N L O\{m\}}=\int_{m}\left[d \sigma^{V}+\int_{1} d \sigma^{A}\right]_{\epsilon=0}$.

Performing the analytic integration $\int_{1} d \sigma^{A}$, one obtains $\epsilon$-pole contributions that can be combined with those in $d \sigma^{V}$, thus cancelling all the divergences. The remainder is finite in the limit $\epsilon \rightarrow 0$ and thus defines the integrand of a cross section contribution $\sigma^{N L O}\{m\}$ with $m$-parton kinematics that can be integrated numerically in four dimensions.

The final structure of the NLO calculation is as follows

$\sigma^{N L O}=\sigma^{N L O\{m+1\}}+\sigma^{N L O\{m\}}$,

and can be easily implemented in a "partonic Monte Carlo' program, which generates appropriately weighted partonic events with $m+1$ finalstate partons and events with $m$ partons.
Note that, using the subtraction method, no approximation is actually performed in the evaluation of the NLO cross section. Rather than approximating the cross section, the subtracted contribution $d \sigma^{A}$ defines a fake cross section that has the same dynamical singularities as the real one and whose kinematics are sufficiently simple to permit its analytic integration.

The real cross section contribution $d \sigma^{R}$ has the following general structure

$$
d \sigma^{R}=d \Phi^{(m+1)}\left|\mathcal{M}_{m+1}\left(\left\{p_{k}\right\}\right)\right|^{2} F_{J}^{(m+1)}\left(\left\{p_{k}\right\}\right),
$$

where $d \Phi^{(m+1)}$ and $\left|\mathcal{M}_{m+1}\right|^{2}$ depend on the process and $F_{J}^{(m+1)}$ depends on the quantity we want to compute. Obviously, for any given $d \sigma^{R}$ one can try to construct a corresponding $d \sigma^{A}$ by properly approximating $d \Phi^{(m+1)},\left|\mathcal{M}_{m+1}\right|^{2}$ and $F_{J}^{(m+1)}$. It is less obvious that one can use the subtraction method to compute arbitrary quantities in a given process, because one needs a fake cross section $d \sigma^{A}$ that depends only on the process and, hence, is independent of the actual definition of the jet function $F_{J}^{(m+1)}$. It is still less obvious that one can use the subtraction method to construct a universal algorithm for computing arbitrary quantities in arbitrary processes. To this purpose the fake cross section $d \sigma^{A}$ also has to be somehow independent of $\mathcal{M}_{m+1}$.

Our method to achieve this generality is based on the dipole formalism.

\section{DIPOLE FORMALISM AND UNI- VERSAL SUBTRACTION TERM}

\subsection{Soft and collinear limits}

The starting point of the dipole formalism are the soft and collinear factorization theorems for the QCD matrix elements. According to these theorems, the singular behaviour in $d$ dimensions of a generic tree-level matrix element $\mathcal{M}_{m+1}\left(p_{1}, \ldots, p_{m+1}\right)$ with $m+1$ final-state partons can be obtained by means of factorized limiting formulae that, respectively in the soft (when the parton momentum $p_{j}$ vanishes) and collinear (when the parton momenta $p_{i}$ and $p_{j}$ become par- 
allel) regions, have the following structure

$$
\begin{aligned}
& \left|\mathcal{M}_{m+1}\left(p_{1}, \ldots, p_{j}, \ldots, p_{m+1}\right)\right|^{2} \rightarrow \\
& \left|\mathcal{M}_{m}\left(p_{1}, \ldots, p_{m+1}\right)\right|^{2} \otimes_{c} \boldsymbol{J}^{2}\left(p_{j}\right) \\
& \left|\mathcal{M}_{m+1}\left(p_{1}, \ldots, p_{j}, p_{i}, \ldots, p_{m+1}\right)\right|^{2} \rightarrow \\
& \left|\mathcal{M}_{m}\left(p_{1}, \ldots, p_{j}+p_{i}, \ldots, p_{m+1}\right)\right|^{2} \otimes_{h} P_{i j}
\end{aligned}
$$

The notation in Eqs. (11,12) is symbolic (see Ref. [6] for more details) but sufficient to recall their main features.

The contributions $\mathcal{M}_{m}$ on the right-hand sides are the tree-level matrix elements to produce $m$ partons and are respectively obtained from the original $m+1$-parton matrix element by removing the soft parton $p_{j}$ or combining the two collinear partons $p_{j}$ and $p_{i}$ into a single-parton momentum.

The other contributions on the right-hand sides are responsible for the soft and collinear divergences. The factor $\boldsymbol{J}^{2}\left(p_{j}\right)$ in Eq. (11) is the eikonal current for the emission of the soft gluon $p_{j}$, and $P_{i j}$ is the Altarelli-Parisi splitting function. These factors are universal: they do not depend on the process but only on the momenta and quantum numbers of the QCD partons in $\mathcal{M}_{m}$. In particular, $\boldsymbol{J}^{2}\left(p_{j}\right)$ depends on the colour charges of the partons in $\mathcal{M}_{m}$, and $P_{i j}$ depends on their helicities. Because of these colour and helicity correlations (symbolically denoted by $\otimes_{c}$ and $\otimes_{h}$ ), Eqs. (11, 12 are not real factorized expressions. Moreover, there is another important reason, due to kinematics, why Eqs. (11,12) cannot be regarded as true factorization formulae but rather as limiting formulae. Indeed, the tree-level matrix elements in Eqs. (11,12) are unambiguously defined only when momentum conservation is fulfilled exactly. Since, in general, the $m+1$ parton phase space does not factorize into an $m$-parton times a single-parton phase space, the right-hand sides of these equations are unequivocally defined only in the strict soft and collinear limits.

Owing to their universality, the limiting formulae (11] 12) can be used to approximate the matrix element $\left|\mathcal{M}_{m+1}\right|^{2}$ in Eq. (10) and thus to find a subtracted cross section $d \sigma^{A}$ that matches the real cross section $d \sigma^{R}$ in all the singular regions of phase space. However, the implementation of Eqs. (11, 12) in the calculation of QCD cross sections requires a careful treatment of momentum conservation away from the soft and collinear limits. Care also has to be taken to avoid double counting the soft and collinear divergences in their overlapping region (e.g. when $p_{j}$ is both soft and collinear to $p_{i}$ ). The use of the dipole factorization theorem introduced in Ref. [21] allows one to overcome these difficulties in a straightforward way.

\subsection{Dipole formulae}

The dipole factorization formulae have the following symbolic structure

$$
\begin{aligned}
& \left|\mathcal{M}_{m+1}\left(p_{1}, \ldots, p_{m+1}\right)\right|^{2}= \\
& \quad\left|\mathcal{M}_{m}\left(\widetilde{p}_{1}, \ldots, \widetilde{p}_{m}\right)\right|^{2} \otimes \boldsymbol{V}_{i j}+\ldots .
\end{aligned}
$$

The dots on the right-hand side stand for contributions that are not singular when $p_{i} \cdot p_{j} \rightarrow 0$. The dipole splitting functions $\boldsymbol{V}_{i j}$ are universal (process-independent) singular factors that depend on the momenta and quantum numbers of the $m$ partons in the tree-level matrix element $\left|\mathcal{M}_{m}\right|^{2}$. Colour and helicity correlations are denoted by the symbol $\otimes$. The set $\widetilde{p}_{1}, \ldots, \widetilde{p}_{m}$ of modified momenta on the right-hand side of Eq. (13) is defined starting from the original $m+1$ parton momenta in such a way that the $m$ partons in $\left|\mathcal{M}_{m}\right|^{2}$ are physical, that is, they are on-shell and energy-momentum conservation is implemented exactly:

$\widetilde{p}_{i}^{2}=0, \quad \widetilde{p}_{1}+\ldots+\widetilde{p}_{m}=p_{1}+\ldots+p_{m+1}$.

The detailed expressions for these parton momenta and for the dipole splitting functions are given in Ref. [6].

Apart from the presence of colour and helicity correlations, Eq. (13) can be considered as a true factorization formula because its left-hand and right-hand sides live on the same phase-space manifold. Equation (14) indeed guarantees that exact kinematics are retained in the definition of the $m$-parton configuration $\left\{\widetilde{p}_{1}, \ldots, \widetilde{p}_{m}\right\}$. These $m$ parton momenta depend on $p_{i}$ and $p_{j}$ in such a way that in the soft and collinear regions the $m$-parton configuration become indistinguishable from the original $m+1$-parton configuration. Correspondingly, the dipole splitting function $\mathbf{V}_{i j}$ is 
defined in order to coincide with the eikonal current and with the Altarelli-Parisi splitting function respectively in the soft and collinear limits.

It follows that Eq. (13) provides a single formula that approximates the real matrix element $\left|\mathcal{M}_{m+1}\right|^{2}$ for an arbitrary process, in all of its singular limits. These limits are approached smoothly, thus avoiding double counting of overlapping soft and collinear singularities. The exact implementation of momentum conservation makes possible this smooth transition and the extrapolation of the limiting formulae (11, 12) away from the soft and collinear regions.

\subsection{Universal subtraction term}

These main features of the dipole formulae allow us to construct a universal subtraction term with the following form

$$
\begin{aligned}
d \sigma^{A}= & d \Phi^{(m+1)} \sum_{i j}\left|\mathcal{M}_{m}\left(\left\{\widetilde{p}_{k}\right\}\right)\right|^{2} \otimes \boldsymbol{V}_{i j} \\
& \cdot F_{J}^{(m)}\left(\left\{\widetilde{p}_{k}\right\}\right) .
\end{aligned}
$$

Note that the only dependence on the jet observable is in the jet-defining function $F_{J}^{(m)}$ and the only dependence on the process is in the treelevel matrix element $\left|\mathcal{M}_{m}\right|^{2}$. These are the same $m$-parton functions as enter in the calculation of the Born-level cross section of Eq. (3). The only other ingredients needed to construct $d \sigma^{A}$ are the dipole splitting functions, which are completely process-independent and given once and for all [6]. This specifies the universal character of Eq. (15): the fake cross section $d \sigma^{A}$ used for the NLO calculation is straightforwardly obtained in terms of the sole (process-dependent) information that is necessary for the corresponding LO calculation.

Having the subtraction term in the explicit form (15), we can discuss how it fulfils the properties $i$ ) and $i i$ ) listed in Sect. 3. As for the property $i$ ), we note that there are several dipole terms on the right-hand side of Eq. (15). Each of them mimics one of the $m+1$-parton configurations in $d \sigma^{R}$ that are kinematically degenerate with a given $m$-parton state. Any time the $m+1$-parton state in $d \sigma^{R}$ approaches a soft and/or collinear region, there is a corresponding dipole factor in $d \sigma^{A}$ that approaches the same region with exactly the same probability as in $d \sigma^{R}$. The equality of the two probabilities directly follows from (15) and from the limiting behaviour in Eqs. (4.11, 12) of the cross section factors on the right-hand side of Eq. (10). In this manner $d \sigma^{A}$ acts as a local counterterm for $d \sigma^{R}$. Note, in particular, that the cancellation mechanism is completely independent of the actual form of the jet-defining function and works for any jet observable (i.e. for any quantity that fulfils Eq. (4)) .

As for the property $i i$ ), we start by noting that $d \sigma^{A}$ (likewise $d \sigma^{R}$ ) depends on the $m+1$ parton momenta $p_{1}, \ldots, p_{m+1}$. However, having introduced the modified momenta $\widetilde{p}_{1}, \ldots, \widetilde{p}_{m}$, for each dipole term in Eq. (15) we can define a one-to-one mapping

$\left\{p_{1}, \ldots, p_{m+1}\right\} \leftrightarrow\left\{\widetilde{p}_{1}, \ldots, \widetilde{p}_{m}, p_{i}+p_{j}\right\}$.

The key feature of this mapping is that the $m$ modified momenta can be chosen in such a way that they obey exact phase-space factorization as follows

$$
\begin{aligned}
d \Phi^{(m+1)}\left(p_{1}, \ldots, p_{m+1}\right)= & d \Phi^{(m)}\left(\widetilde{p}_{1}, \ldots, \widetilde{p}_{m}\right) \\
& \cdot d \varphi_{\left(\left\{\widetilde{p}_{k}\right\}\right)}\left(p_{i}+p_{j}\right),
\end{aligned}
$$

where $d \varphi$ is a single-particle subspace that, for fixed $\widetilde{p}_{1}, \ldots, \widetilde{p}_{m}$, depends only on the dipole momenta $p_{i}$ and $p_{j}$ [6]. Owing to the exact phasespace factorization and to the fact that the fake cross section in Eq. (15) is proportional to the jet quantity calculated from the modified $m$ parton configuration, the integration of the singular dipole contributions can be completely factorized (modulo colour and helicity correlations) with respect to a term that exactly reproduces the Born-level cross section:

$$
\begin{aligned}
\int_{m+1} d \sigma^{A} & =\int_{m} d \Phi^{(m)}\left(\left\{\widetilde{p}_{k}\right\}\right)\left|\mathcal{M}_{m}\left(\left\{\widetilde{p}_{k}\right\}\right)\right|^{2} \\
\cdot F_{J}^{(m)}\left(\left\{\widetilde{p}_{k}\right\}\right) & \otimes \sum_{i j} \int_{1} d \varphi_{\left(\left\{\widetilde{p}_{k}\right\}\right)}\left(p_{i}+p_{j}\right) \boldsymbol{V}_{i j} \\
= & \int_{m} d \sigma^{B} \otimes \boldsymbol{I}\left(\left\{\widetilde{p}_{k}\right\}\right) .
\end{aligned}
$$

The last factor on the right-hand side of Eq. (18) is defined by

$$
\boldsymbol{I}\left(\left\{\widetilde{p}_{k}\right\}\right) \equiv \sum_{i j} \int_{1} d \varphi_{\left(\left\{\widetilde{p}_{k}\right\}\right)}\left(p_{i}+p_{j}\right) \boldsymbol{V}_{i j}
$$


and contains all the soft and collinear singularities that are necessary to compensate those in the virtual cross section $d \sigma^{V}$. Owing to the convenient definition of the dipole splitting function $\boldsymbol{V}_{i j}$, it is possible to carry out analytically the integration in Eq. (19) over the dipole phase space in $d$ dimensions. This leads to an explicit and universal expression [6] for the factor $\boldsymbol{I}$, whose $\epsilon$-poles cancel those in the one-loop matrix element.

\section{FINAL RESULTS AND NUMERICAL IMPLEMENTATION}

The discussion in the previous section shows that, by using the subtraction method and the dipole formulae, one can extract and treat analytically the singular parts of a NLO cross section in a way that is independent of the exact details of the observable and of the process. This leaves a remainder that depends on the full complications of the jet quantity, but which is finite so that it can be treated either numerically or analytically (whenever possible).

In general, the use of numerical integration techniques (typically, Monte Carlo methods) is certainly more convenient. First of all, the numerical approach allows one to calculate any number and any type of observable simultaneously by simply histogramming the appropriate quantities, rather than having to make a separate analytic calculation for each observable. Furthermore, using the numerical approach, it is easy to implement different experimental conditions, for example detector acceptances and experimental cuts.

In order to summarize the final results of our algorithm and to describe their numerical implementation, we start by recalling how the LO cross section in Eq. (2) is evaluated by using a Monte Carlo program. One first generates an $m$-parton event in the phase-space region $d \Phi^{(m)}$ and gives it the weight $\left|\mathcal{M}_{m}\right|^{2}$. Then this weighted event is analysed by a user routine according to the actual definition of the phase space function $F_{J}^{(m)}$ and inserted into a corresponding histogram bin.

Following the decomposition in Eq. (9), the NLO cross section is obtained by adding two contributions (which are not necessarily positive def- inite) with $m$-parton (as in the LO calculation) and $m+1$-parton kinematics, respectively. Unlike the original real and virtual contributions, these two terms are separately finite and can be directly integrated in four space-time dimensions.

The first contribution is obtained by inserting Eq. (18) into Eq. (8) and is explicitly given by

$$
\begin{aligned}
& \sigma^{N L O\{m\}}=\int_{m} d \Phi^{(m)} F_{J}^{(m)}\left(\left\{p_{k}\right\}\right) \\
& \cdot\left\{\left|\mathcal{M}_{m}\left(\left\{p_{k}\right\}\right)\right|_{(1-l o o p)}^{2}\right. \\
& \left.+\left|\mathcal{M}_{m}\left(\left\{p_{k}\right\}\right)\right|^{2} \otimes \boldsymbol{I}\left(\left\{p_{k}\right\}\right)\right\}_{\epsilon=0} .
\end{aligned}
$$

The first term in the curly bracket is the oneloop renormalized matrix element for producing $m$ final-state partons. The second term is obtained by combining the tree-level matrix element to produce $m$ partons and the universal factor I in Eq. (19). These two terms are defined in $d=4-2 \epsilon$ dimensions. Great progress has been made in recent years in the analytical techniques for evaluating loop amplitudes, and many of them have been calculated [22, 团. The explicit expression of the universal factor $\mathbf{I}$ is provided by our algorithm. Thus, one has to carry out the expansion in $\epsilon$-poles of the two terms in the curly bracket, cancel analytically (by trivial addition) the poles and perform the limit $\epsilon \rightarrow 0$. This simple algebraic manipulation is sufficient to construct an effective $m$-parton weight (the curlybracket contribution on the right-hand side) that is finite. As a result, Eq. (20) can be handled by the Monte Carlo program exactly in the same way as the LO cross section.

The NLO contribution with $m+1$-parton kinematics, which is obtained by subtracting the fake cross section in Eq. 15) from the real cross section in Eq. (10), has the following explicit expression:

$$
\begin{aligned}
& \sigma^{N L O\{m+1\}}=\int_{m+1} d \Phi^{(m+1)} \\
& \cdot\left\{\left|\mathcal{M}_{m+1}\left(\left\{p_{k}\right\}\right)\right|^{2} F_{J}^{(m+1)}\left(\left\{p_{k}\right\}\right)\right. \\
& \left.-\sum_{i j}\left|\mathcal{M}_{m}\left(\left\{\widetilde{p}_{k}\right\}\right)\right|^{2} \otimes \boldsymbol{V}_{i j} F_{J}^{(m)}\left(\left\{\widetilde{p}_{k}\right\}\right)\right\},
\end{aligned}
$$

The terms in the curly bracket define an effective 
matrix element that is integrable in four spacetime dimensions. It follows that the NLO matrix element $\mathcal{M}_{m+1}$, with $m+1$ final-state partons, can be directly evaluated in $d=4$ dimensions thus leading to an extreme simplification of the Lorentz algebra. Knowing the tree-level matrix elements and the dipole splitting functions, the Monte Carlo integration of Eq. (21) is straightforward. One simply generates an $m+1$-parton configuration and uses it to define an event with positive weight $+\left|\mathcal{M}_{m+1}\right|^{2}$ and several counterevents, each of them with the negative weight $-\left|\mathcal{M}_{m}\right|^{2} \otimes \mathbf{V}_{i j}$. Then these event and counterevents are analysed by the user routine. The role of the two different jet functions $F_{J}^{(m+1)}$ and $F_{J}^{(m)}$ is that of binning the weighted event and counterevents into different bins of the jet observable. Any time that the generated $m+1$-parton configuration approaches a singular region, the event and one counter-event fall into the same bin and the cancellation of the large positive and negative weights takes place.

\section{MONTE CARLO PROGRAMS}

Generalizing the procedure for constructing NLO Monte Carlo programs for arbitrary quantities has several advantages. These are principally due to the reduction in the number and complexity of ingredients that have to be calculated for each new process, and because the $d$-dimensional integrals only need be done once and can be easily checked independently, rather than being buried inside a specific calculation.

Using the general algorithm described in this contribution, we have already constructed a Monte Carlo program (EVENT2) for three-jet observables in $e^{+} e^{-}$annihilation [21]. In the case of un-oriented three-jet events, this program is comparable and in agreement with the program EVENT [23], which uses the subtraction procedure introduced in Ref. 10]. In the case of oriented events, our program 24 should be compared with a corresponding program, EERAD [12], based on the phase-space slicing method.

A NLO program for $2+1$-jet observables in deep-inelastic lepton-hadron scattering will soon be available [25] and compared with the Monte
Carlo MEPJET [26] that implements the phasespace slicing algorithm of Ref. [13].

\section{SUMMARY AND OUTLOOK}

The calculation of jet cross sections in perturbative QCD requires the integration of multiparton matrix elements over complicated phasespace regions that depend on the actual definition of the jet observables and on the experimental cuts. In general, these phase-space integrations can be carried out only by using numerical methods. Beyond LO, however, numerical techniques cannot be straightforwardly applied because realemission contributions and virtual contributions are separately divergent. These divergences have to be first regularized, then evaluated analytically, combined together and cancelled before any numerical calculation can be attempted.

The algorithm described in this contribution overcomes in a simple way all the analytical difficulties related to the treatment of soft and collinear divergences in NLO calculations. Using the subtraction method and the dipole formulae, we are able to explicitly carry out all the analytical work that is necessary to evaluate and cancel the singularities. The final output of the algorithm is given in terms of effective matrix elements that can be automatically constructed, starting from the original (process-dependent) matrix elements and universal (process-independent) dipole factors. The effective matrix elements can be numerically or analytically (whenever possible) integrated over the available phase space in four dimensions to compute the actual value of the NLO cross section. If the numerical approach is chosen, Monte Carlo integration techniques can be easily implemented to provide a general-purpose Monte Carlo program for carrying out NLO QCD calculations in any given process.

The simplified discussion of the algorithm presented in this contribution directly applies to processes, like $e^{+} e^{-} \rightarrow n$ jets, in which there are neither initial-state hadrons nor identified hadrons in the final state. However, the formalism and the algorithm are completely general in the sense that they apply to any jet observable 
in a given scattering process as well as to any hard-scattering process. Full details and explicit results for lepton-hadron and hadron-hadron collisions and for fragmentation processes are given in Ref. 6. The inclusion of heavy quarks in the algorithm can be performed in a completely general and process-independent manner and will be presented elsewhere. Another potentially important extension is the generalization to polarized scattering processes, which is also straightforward in the dipole formalism.

At present, QCD calculations to next-to-nextto-leading order (NNLO) are available only for some fully inclusive quantities (see Ref. [28] and references therein). In this case one considers all possible final states and integrates the QCD matrix elements over the whole final-state phase space. Thus one can add real and virtual contributions before performing the relevant momentum integrations in such a way that only ultraviolet singularities appear at the intermediate steps of the calculation. In the case of less inclusive jet observables, one cannot take advantage of the cancellation of soft and collinear divergences at the integrand level and, at present, no straightforward method is available to handle these divergences at NNLO. Even once the necessary twoloop matrix elements for several processes are calculated, the amount of work needed to provide a numerical implementation will be enormous. The main features of the dipole formalism, which permit a universal treatment of soft and collinear singularities at NLO, seem particularly suited to set up a general method for carrying out NNLO QCD calculations.

Acknowledgements. We would like to thank J. Bluemlein, F. Jegerlehner and T. Riemann for the excellent organization of a very interesting Workshop.

\section{REFERENCES}

1. S. Catani, in Proc. of the Int. Europhysics Conf. on High Energy Physics, HEP 93, eds. J. Carr and M. Perrottet (Editions Frontières, Gif-sur-Yvette, 1994), p. 771; B.R. Webber, in Proc. of the 27th Int. Conf. on High Energy
Physics, eds. P.J. Bussey and I.G. Knowles (Institute of Physics, Bristol, 1995), p. 213.

2. W.T. Giele, these Proceedings.

3. V. Braun, these Proceedings; G. Marchesini, these Proceedings.

4. D.A. Kosower, these Proceedings.

5. G. Marchesini, B.R. Webber, G. Abbiendi, I.G. Knowles, M.H. Seymour and L. Stanco, Comput. Phys. Commun. 67 (1992) 465.

6. S. Catani and M.H. Seymour, preprint CERN-TH/96-29 (hep-ph/9605323).

7. P. de Causmaecker, R. Gastmans, W. Troost and T.T. Wu, Phys. Lett. 105B (1981) 215; R. Kleiss, Nucl. Phys. B241 (1984) 61; F.A. Berends, P.H. Daverveldt and R. Kleiss, Nucl. Phys. B253 (1985) 441; J.F. Gunion and Z. Kunszt, Phys. Lett. 161B (1985) 333; Z. Xu, D.H. Zhang and L. Chang, Nucl. Phys. B291 (1987) 392.

8. M.L. Mangano and S.J. Parke, Phys. Rep. 200 (1991) 301, and references therein.

9. K. Fabricius, G. Kramer, G. Schierholz and I. Schmitt, Z. Phys. C11 (1981) 315; G. Kramer and B. Lampe, Fortschr. Phys. 37 (1989) 161.

10. R.K. Ellis, D.A. Ross and A.E. Terrano, Nucl. Phys. B178 (1981) 421.

11. See, for instance: A. Bassetto, M. Ciafaloni and G. Marchesini, Phys. Rep. 100 (1983) 201; Yu.L. Dokshitzer, V.A. Khoze, A.H. Mueller and S.I. Troyan, Basics of Perturbative QCD, Editions Frontières, Paris, 1991.

12. W.T. Giele and E.W.N. Glover, Phys. Rev. D 46 (1992) 1980.

13. W.T. Giele, E.W.N. Glover and D.A. Kosower, Nucl. Phys. B403 (1993) 633; W.T. Giele, these Proceedings.

14. H. Baer, J. Ohnemus and J.F. Owens, Phys. Rev. D 42 (1990) 61; B. Bailey, J.F. Owens and J. Ohnemus, Phys. Rev. D 46 (1992) 2018.

15. W.T. Giele, S. Keller and E. Laenen, Phys. Lett. 372B (1996) 141; S. Keller and E. Laenen, preprint in preparation.

16. Z. Kunszt and D.E. Soper, Phys. Rev. D 46 (1992) 192.

17. S. Frixione, Z. Kunszt and A. Signer, Nucl. Phys. B467 (1996) 399.

18. M.L. Mangano, P. Nason and G. Ridolfi, 
Nucl. Phys. B373 (1992) 295. S. Frixione, M.L. Mangano, P. Nason and G. Ridolfi, Nucl. Phys. B412 (1994) 225.

19. Z. Nagy and Z. Trócsányi, preprint KLTEDTP/96-1 (February 1996).

20. Z. Kunszt, these Proceedings.

21. S. Catani and M.H. Seymour, Phys. Lett. 378B (1996) 287.

22. Z. Bern, L. Dixon, D.C. Dunbar and D.A. Kosower, Nucl. Phys. B425 (1994) 217; Z. Kunszt, A. Signer and Z. Trócsányi, Phys. Lett. 336B (1994) 529; Z. Bern, L. Dixon and D.A. Kosower, preprint SLAC-PUB-7111 (hep-ph/9602280).
23. Z. Kunszt and P. Nason, in ' Z Physics at LEP 1', CERN 89-08, vol. 1, p. 373.

24. P. Nason and B.R. Webber, conveners, $Q C D$, in 'Physics at LEP2', CERN 96-01, vol. 1, p. 249 (hep-ph/9602288).

25. S. Catani and M.H. Seymour, in preparation.

26. E. Mirkes, these Proceedings; E. Mirkes and D. Zeppenfeld, preprint MADPH-95-916 (hep-ph/9511448).

27. S. Catani and M.H. Seymour, in preparation. 28. K. Chetyrkin, these Proceedings; S. Larin, these Proceedings; J. Vermaseren, these Proceedings. 